\title{
Treatment of hemiballismus with stereotactic pallidotomy
}

\author{
Case report and review of the literature
}

\author{
Konstantin V. Slavin, M.D., Thomas K. Baumann, Ph.D., And Kim J. Burchiel, M.D. \\ Department of Neurological Surgery, Oregon Health Sciences University, Portland, Oregon
}

\begin{abstract}
Hemiballismus is a relatively rare movement disorder that is characterized by uncontrolled, random, large-amplitude movements of the limbs. It is usually caused by a vascular lesion that involves the contralateral subthalamic nucleus (STN) (also known as the nucleus hypothalamicus or corpus luysi) and its afferent and efferent pathways.

The authors present a case of medically intractable hemiballismus in a 70-year-old woman who was successfully treated with stereotactic posteroventral pallidotomy. In agreement with the data reported earlier by other groups, the microrecording performed during the pallidotomy showed a decreased rate of firing of the pallidal neurons, supporting the theory of impaired excitatory input from the STN to the internal part of the globus pallidus.

Stereotactic pallidotomy may be the procedure of choice in the treatment of medically intractable hemiballismus. Intraoperative microrecording significantly improves the precision of the stereotactic targeting and should be considered a standard part of the pallidotomy protocol.
\end{abstract}

KEY WORDS • hemiballismus • pallidotomy - stereotactic neurosurgery

Hemiballismus is a peculiar hyperkinetic disorder that appears as irregular and uncoordinated contorting movements of one half of the body, with a pronounced tendency to twist and turn..$^{50}$ These movements are involuntary, violent, and poorly patterned. ${ }^{25}$ The syndrome of hemiballismus develops most commonly in patients with vascular or other injury of the STN and its connections. Despite the fact that hemiballismus is usually self-limiting and responsive to conservative treatment with neuroleptic, sedative, and other neuroactive medications, some patients with intractable disease are referred to neurosurgeons for surgical intervention.

We present a case of medically intractable hemiballismus that was successfully treated with stereotactic posteroventral pallidotomy. In agreement with the data reported earlier by other groups, ${ }^{43,49}$ the microrecording performed during the pallidotomy showed a decreased rate of firing of the pallidal neurons, supporting the theory of impaired excitatory input from the STN to the GP. ${ }^{1,11,15,18}$

\section{CASE REPORT}

History. This 70-year old woman with a history of hypertension and insulin-dependent diabetes mellitus presented with new onset of uncontrolled violent movements of the left extremities accompanied by headaches. Her symptoms started for no obvious reason; the move-

Abbreviations used in this paper: $\mathrm{CT}=$ computerized tomography; GP = globus pallidus; GPi = GP internus; PD = Parkinson disease; $\mathrm{STN}=$ subthalamic nucleus. ments were more pronounced in the arm than in the leg, and their amplitude increased over a period of 2 to 3 days after onset.

Examination. The patient was in good health on admission and results of her neurological examination were essentially normal except for the constant, relentless, violent movement of her left arm and leg. Although she was fully awake, alert, and oriented, she could not stop the abnormal movements except for a short period of time, and even then a major effort was required. Despite the protective cushioning that was applied to her bed to prevent injuries, both her left arm and leg were covered with bruises and lacerations. Attempts to restrain her from the flinging her extremities were also unsuccessful. The movements on the right side were unremarkable, but the patient could not perform any normal activity because of the left hemiballismus. All abnormal movements always stopped when she was asleep.

Admission CT scans of the brain were obtained and revealed hypodensities in the right STN and the posterior limb of the left internal capsule (Fig. 1). Based on the clinical picture and CT appearance, we diagnosed a stroke involving the right STN.

Initial Treatment. The patient's medical treatment consisted of a combination of neuroleptic agents (haloperi$\mathrm{dol}, \leq 10 \mathrm{mg} /$ day) and benzodiazepines (lorazepam, $\leq 15$ $\mathrm{mg} /$ day). This regimen only minimally decreased the abnormal movements but made the patient very somnolent. Chlorpromazine was also tried, but led to only a mild decrease in the frequency of ballistic movement. The patient's condition started to deteriorate because of severe 


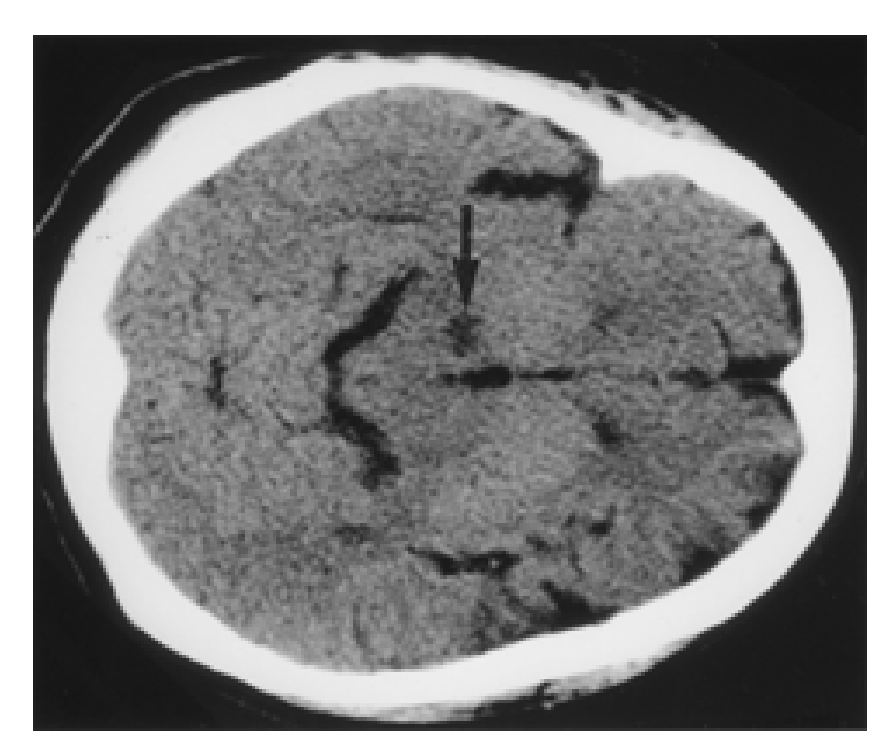

Fig. 1. Nonenhanced axial CT scan demonstrating a hypodensity in the projection of the right STN (arrow).

exhaustion. She also required a feeding tube to get some nourishment. Surgery was recommended after the 10-day trial of conservative management failed.

Operation. To prepare her for the pallidotomy, anesthesia was induced and the patient was intubated. A Leksell frame was applied, and the stereotactic magnetic resonance images were obtained using the standard protocol for pallidotomy. Axial $\mathrm{T}_{1}$-weighted, axial inversion-recovery, and coronal fast-spin echo magnetic resonance images were obtained. The GP, the internal capsule, and the optic tract were identified on both sides, and after that the stereotactic coordinates for the target point were calculated. The target was chosen at $19 \mathrm{~mm}$ to the right of the midline, $2 \mathrm{~mm}$ anterior to the midcommissural point, and $6 \mathrm{~mm}$ inferior to the plane of the anterior commissureposterior commissure.

After targeting was completed, the cannula was inserted through the twist-drill opening in the right precoronal region parallel to the sagittal plane and at a $65^{\circ}$ angle to the axial plane. Microelectrode recording ${ }^{44,45}$ was used to locate the GP externus, the internal medullary lamina, the $\mathrm{GPi}$, and the optic tract. A total of six neurons was recorded, two in the GP externus and four in the GPi. The firing rate and pattern of the GPi neurons were somewhat different compared with those observed in patients with PD and patients with dystonias. The mean firing rate, for example, was $35 \mathrm{~Hz}$ as opposed to the mean GPi firing rate of $85 \mathrm{~Hz}$ described in the literature (Fig. 2). The kinesthetic response was elicited with passive movement of the left arm. The optic tract location was confirmed by stroboscopic stimulation. After completion of the microrecording, the standard method of radiofrequency thermocoagulation was used after heating the tip of the probe to $84^{\circ} \mathrm{C}$ for 60 seconds. A standard 1.1-mm-diameter probe with a 2-mm bare tip was used for the pallidotomy. Three lesions were made: one at the target point, one at $2 \mathrm{~mm}$, and one at $4 \mathrm{~mm}$ superior to it along the trajectory of the electrode, and then the electrode and the cannula were removed.
Postoperative Course. The patient exhibited no change in the severity of her ballistic movements immediately after the surgery. Over the next 24 hours, however, there was a gradual decrease in both amplitude and frequency of the abnormal movements. The patient maintained normal strength in all extremities and regained voluntary control over movements on the left side.

At the follow-up examination 6 months postoperatively, the patient had returned to her normal activities, was able to walk without assistance, and all her bruises and abrasions gradually healed. She continued to have lowamplitude choreiform involuntary movements in the left leg, but this did not limit her functioning. More detailed examination revealed normal cranial nerves, no visual deficits, mild (4+/5) weakness in the left arm, and some disturbance of the gait with short steps and wide base. The walking problem had improved with physical therapy.

\section{DISCUSSION}

Hemiballismus is a relatively rare movement disorder that is characterized by uncontrolled, random, large-amplitude movements of the limbs; these movements are usually most pronounced in the proximal joints. The movements in hemiballismus are limited to one side of the body, and they may also involve axial musculature, face, and tongue. The pathological finding most commonly encountered in cases of hemiballismus is a vascular lesion that involves the contralateral STN (also known as the nucleus hypothalamicus or corpus luysi) and its afferent and efferent pathways. ${ }^{31,35,50}$ Other structural lesions, such as neoplasms, ${ }^{27}$ tuberculomas,${ }^{50}$ and hemorrhages ${ }^{31,50}$ as well as lesions in other areas, such as the contralateral cerebral cortex, caudate nucleus, thalamus, striatum, internal capsule, ${ }^{5,26}$ and ipsilateral STN $^{10}$ have been described as causative factors of hemiballismus. Metabolic and systemic abnormalities including thyrotoxicosis, hyperglycemia, lupus, and scleroderma have also been reported as causes of hemiballismus. ${ }^{29,36}$ Hemiballismus was also encountered in patients who had suffered head trauma ${ }^{8,36}$ and after they had undergone thalamotomies and subthalamotomies for tremor control. $4,13,16,24,34$

In the past, the prognosis for hemiballismus was uniformly grave, ${ }^{33,50}$ and this fact prompted development of various surgical procedures for control of the disease. The operations proposed for this purpose included limb amputation and brachial plexus destruction, ${ }^{33,38}$ cortical resection, ${ }^{2,7,33}$ anterior cervical cordotomy, ${ }^{6,42}$ and pedunculotomy. ${ }^{17}$

The natural course of the disease and the effect of various medications were recently studied by multiple investigators; $12,17,23,36,39,41,48$ it appears that in the majority of cases hemiballismus is either self-limiting or may be controlled with orally administered medications, usually neuroleptic and other neuroactive agents. Therefore, surgery is reserved for intractable cases of hemiballismus, after the standard medical treatment fails.

Various stereotactic targets were used for control of abnormal movements associated with hemiballismus, ${ }^{3,40,47}$ but the most consistent success was achieved with ventrolateral thalamotomy ${ }^{8,9,19-21,25,28,32,37}$ and posteroventral pallidotomy. ${ }^{14,25,43,49}$ Chronic thalamic stimulation was also re- 

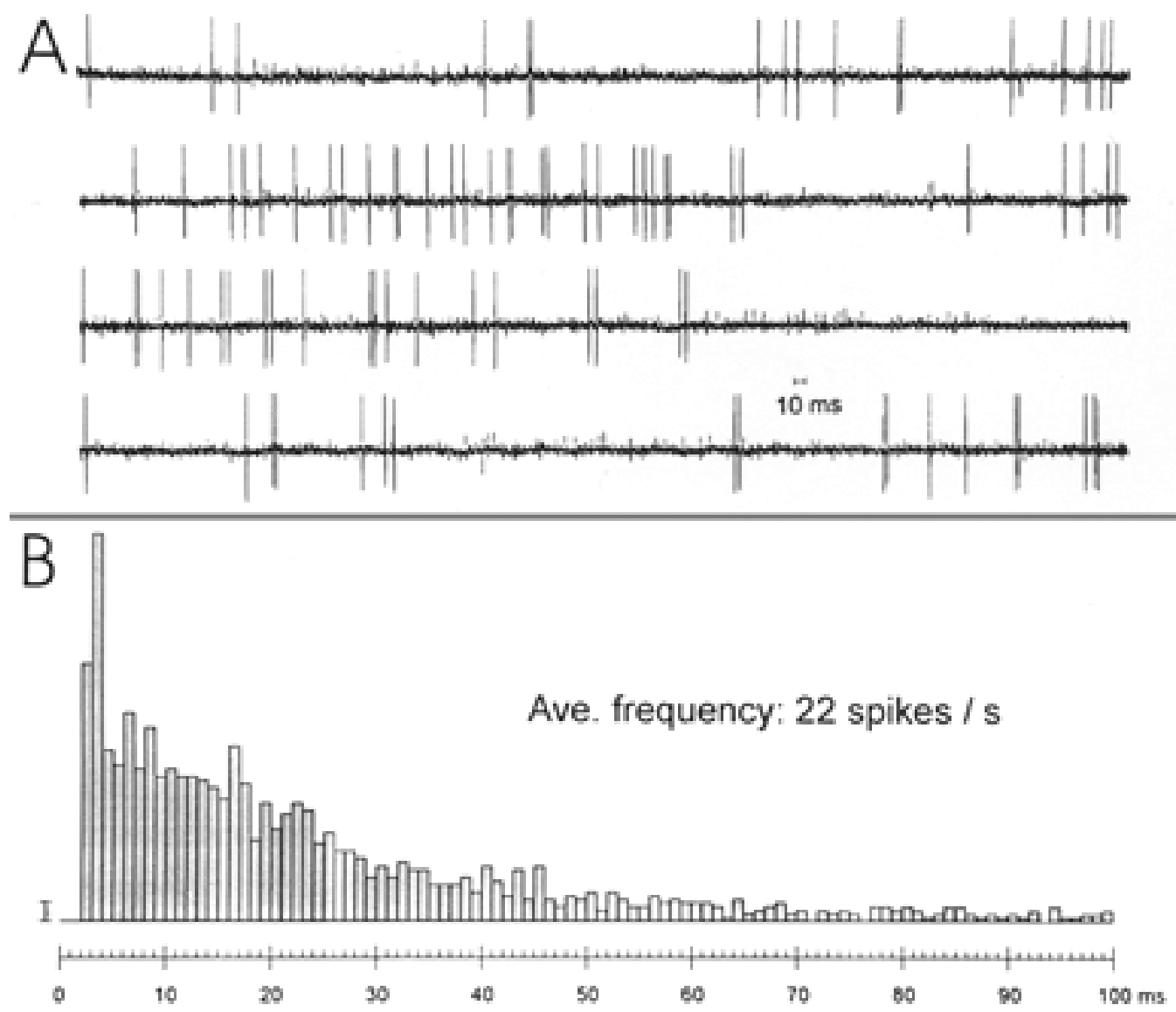

Fig. 2. Microrecordings showing spontaneous discharge of a GPi neuron located less than $0.5 \mathrm{~mm}$ dorsal to the ventral border of the GPi. A: Extracellular recording of action potentials showing that the traces are contiguous. B: Histogram showing interspike intervals for 1590 action potentials collected during 73.1 seconds (same neuron as in A). The mean frequency was $22 \mathrm{~Hz}$. Interspike intervals were as follows: p10, - $4 \mathrm{msec}$; lower quartile (p25), $-8 \mathrm{msec}$; median (p50), - 17 msec; upper quartile (p75); - $32 \mathrm{msec}$; p90, -53 msec. Data were analyzed with the Interspike program written by Jacques Favre, M.D., Ph.D.

ported to be successful in relieving the abnormal movements in hemiballismus. ${ }^{22,46}$

In normal circumstances the STN exhibits excitatory action on the GPi; therefore, one may expect to see pallidal hyperactivity when the STN is disinhibited..$^{1,11,18}$ This situation is observed in cases of PD, in which dopaminergic nigrostriatal pathways that normally inhibit the STN are hypoactive. The hyperactivity of the STN and GPi may be seen on the microelectrode recordings obtained during pallidotomies or subthalamotomies. Suppression of this hyperactive state either by destruction or continuous electrical stimulation of one of these areas or their projective field in the thalamus results in normalization of hypokinetic symptoms and elimination of the tremor in patients with PD; this phenomenon is actively used in contemporary surgical treatment of parkinsonism.

On the other hand, destructive lesioning of the STN will be expected to cause decreased activity in the GPi (with subsequently decreased inhibitory outflow from this structure) and excessive disinhibition of the thalamus, resulting in the uncontrolled movements of the extremities observed in cases of hemiballismus. This theory goes along with the previously documented decreased firing rate in the GPi in patients with hemiballismus, ${ }^{43,49}$ but does not explain the positive effect of further destruction of the GPi by pallidotomy, adding more paradoxes to surgery for movement disorders. ${ }^{30}$ Instead, the rationale for pallidotomy is supported by a theory of patterned GPi activity, which was suggested by Suarez and colleagues ${ }^{43}$ based on the results of pallidal microrecording. According to these authors, hemiballismus is a result of the transmission of patterned output from the GPi to the periphery through thalamocortical projections, and therefore may be eliminated by GPi lesioning.

\section{CONCLUSIONS}

The good outcome after stereotactic pallidotomy in the case presented here once again shows the usefulness of this procedure in the treatment of medically intractable hemiballismus. The precision of the stereotactic targeting is greatly enhanced by intraoperative microrecording, which should be considered a standard part of the pallidotomy protocol.

\section{References}

1. Albin RL, Young AB, Penney JB: The functional anatomy of basal ganglia disorders. Trends Neurosci 12:366-375, 1989 
2. Alpers BJ, Jaeger R: Hemiballism and its control by ablation of the motor cortex. Arch Neurol Psychiatry 64:285-287, 1950

3. Andy OJ: Diencephalic coagualtion in the treatment of hemiballismus. Confin Neurol 22:346-350, 1962

4. Andy OJ, Jurko MF, Sias FR Jr: Subthalamotomy in treatment of parkinsonian tremor. J Neurosurg 20:860-870, 1963

5. Bhatia KP, Marsden CD: The behavioural and motor consequences of focal lesions of the basal ganglia in man. Brain 117: 859-876, 1994

6. Brown MH, Walsh MN: The effect of ventral quadrant section of the cervical cord on hemiballismus. J Neurosurg 11: 409-412, 1954

7. Bucy PC: The surgical treatment of extrapyramidal diseases. J Neurochem 14:108-117, 1951

8. Bullard DE, Nashold BS Jr: Stereotaxic thalamotomy for treatment of posttraumatic movement disorders. J Neurosurg 61: 316-321, 1984

9. Cardoso F, Jankovic J, Grossman RG, et al: Outcome after stereotactic thalamotomy for dystonia and hemiballismus. Neurosurgery 36:501-508, 1995

10. Crozier S, Lehericy S, Verstichel P, et al: Transient hemiballism/hemichorea due to an ipsilateral subthalamic nucleus infarction. Neurology 46:267-268, 1996

11. DeLong MR: Primate models of movement disorders of basal ganglia origin. Trends Neurosci 13:281-285, 1990

12. Dewey RB Jr, Jankovic J: Hemiballism-hemichorea: clinical and pharmacologic findings in 21 patients. Arch Neurol 46: 862-867, 1989

13. Dierssen G, Bergmann LL, Gioino G, et al: Hemiballism following surgery for Parkinson's disease. A clinicoanatomical study of a case. Arch Neurol 5:627-637, 1961

14. Gioino GG, Dierssen G, Cooper IS: The effect of subcortical lesions on production and alleviation of hemiballic and hemichoreic movements. J Neurol Sci 3:10-36, 1966

15. Guridi J, Obeso JA: The role of the subthalamic nucleus in the origin of hemiballism and parkinsonism: new surgical perspectives. Adv Neurol 74:235-247, 1997

16. Hughes B: Involuntary movements following stereotactic operations for parkinsonism with special reference to hemi-chorea (ballismus). J Neurol Neurosurg Psychiatry 28:291-303, 1965

17. Hyland HH, Forman DM: Prognosis in hemiballismus. Neurology 7:381-391, 1957

18. Inzelberg R, Korszyn AD: Hemiballism in Parkinson's disease. J Neurol Neurosurg Psychiatry 58:645-647, 1995 (Letter)

19. Jallo GI, Dogali M: Ventral intermediate thalamotomy for hemiballismus. Stereotact Funct Neurosurg 65:23-25, 1995

20. Jankovic J, Hamilton WJ, Grossman RG: Thalamic surgery for movement disorders. Adv Neurol 74:221-233, 1997

21. Kandel EI: Treatment of hemihyperkinesias by stereotactic operations on basal ganglia. Appl Neurophysiol 45:225-229, 1982

22. Katayama Y, Fukaya C, Yamamoto T: Control of poststroke involuntary and voluntary movement disorders with deep brain or epidural cortical stimulation. Stereotact Funct Neurosurg 69: 73-79, 1997

23. Klawans HL, Moses H III, Nausieda PA, et al: Treatment and prognosis of hemiballismus. N Engl J Med 295:1348-1350, 1976

24. Krauss JK, Mohadjer M, Nobbe F, et al: The treatment of posttraumatic tremor by stereotactic surgery: symptomatic and functional outcome in a series of 35 patients. J Neurosurg 80: 810-819, 1994

25. Krauss JK, Mundinger F: Functional stereotactic surgery for hemiballism. J Neurosurg 85:278-286, 1996

26. Lang AE: Persistent hemiballismus with lesions outside the subthalamic nucleus. Can J Neurol Sci 12:125-128, 1985

27. Lemmen LJ, Davis JS, Fisher ER: Hemiballismus secondary to metastatic carcinoma of the gall bladder. Neurology 7: 873-874, 1957
K. V. Slavin, T. K. Baumann, and K. J. Burchiel

28. Levesque MF, Markham $\mathrm{CH}$ : Ventral intermediate thalamotomy for posttraumatic hemiballismus. Stereotact Funct Neurosurg 58:26-29, 1992

29. Lin JJ, Chang MK: Hemiballism-hemichorea and non-ketotic hyperglycaemia. J Neurol Neurosurg Psychiatry 57: 748-750, 1994

30. Marsden CD, Obeso JA: The functions of the basal ganglia and the paradox of stereotaxic surgery in Parkinson's disease. Brain 117:877-897, 1994

31. Martin JP, Alcock NS: Hemichorea associated with a lesion of the corpus Luysii. Brain 57:504-516, 1934

32. Martin JP, McCaul IR: Acute hemiballismus treated by ventrolateral thalamolysis. Brain 82:104-108, 1959

33. Meyers R, Sweeney DB, Schwidde JT: Hemiballismus: aetiology and surgical treatment. J Neurol Neurosurg Psychiatry 13:115-126, 1950

34. Modesti LM, Van Buren JM: Hemiballismus complicating stereotactic thalamotomy. Appl Neurophysiol 42:267-283, 1979

35. Moersch FP, Kernohan JW: Hemiballismus: a clinicopathologic study. Arch Neurol Psychiatry 41:365-372, 1939

36. Muenter MD: Hemiballismus. Neurology 34 (Suppl 1):129, 1984 (Abstract)

37. Mundinger F, Riechert T, Disselhoff J: Long term results of stereotaxic operations on extrapyramidal hyperkinesia (excluding parkinsonism). Confin Neurol 32:71-78, 1970

38. Schaller WF: Comment on Bucy PC: Case TJ: Athetosis: II. Surgical treatment of unilateral athetosis. Arch Neurol Psychiatry $37: 983-1017,1937$

39. Shannon KM: Hemiballismus. Clin Neuropharmacol 13: 413-425, 1990

40. Spiegel EA, Wycis HT, Szekely EG, et al: Campotomy in various extrapyramidal disorders. J Neurosurg 20:871-884, 1963

41. Stojanovic M, Sternic N, Kostic VS: Clozapine in hemiballismus: report of two cases. Clin Neuropharmacol 20:171-174, 1997

42. Strain RE, Perlmutter I: Hemiballismus relieved by ventral quadrant section of the cervical spinal cord without paralysis. J Neurosurg 14:332-336, 1957

43. Suarez JI, Metman LV, Reich SG, et al: Pallidotomy for hemiballismus: efficacy and characteristics of neuronal activity. Ann Neurol 42:807-811, 1997

44. Taha JM, Favre J, Baumann TK, et al: Functional anatomy of the pallidal base in Parkinson's disease. Neurosurgery 39: 1164-1168, 1996

45. Taha JM, Favre J, Baumann TK, et al: Tremor control after pallidotomy in patients with Parkinson's disease: correlation with microrecording findings. J Neurosurg 86:642-647, 1997

46. Tsubokawa T, Katayama Y, Yamamoto T: Control of persistent hemiballismus by chronic thalamic stimulation. Report of two cases. J Neurosurg 82:501-505, 1995

47. Tsubokawa T, Moriyasu N: Lateral pallidotomy for relief of ballistic movement-its basic evidences and clinical application. Confin Neurol 37:10-15, 1975

48. Vidakovic A, Dragasevic N, Kostic VS: Hemiballism: report of 25 cases. J Neurol Neurosurg Psychiatry 57:945-949, 1994

49. Vitek JL, Kaneoke Y, Hashimoto T, et al: Neuronal activity in the pallidum of a patient with hemiballismus. Ann Neurol 38: 296-297, 1995 (Abstract)

50. Whittier JR: Ballism and the subthalamic nucleus (nucleus hypothalamicus; corpus Luysi). Review of the literature and study of thirty cases. Arch Neurol Psychiatry 58:672-692, 1947

Manuscript received May 27, 2004.

Accepted in final form June 12, 2004.

Address reprint requests to: Konstantin V. Slavin, M.D., Department of Neurosurgery, University of Illinois at Chicago, 912 South Wood Street, M/C 799, Chicago, Illinois 60612. email: kslavin@uic.edu. 